\title{
Developmental demands contribute to early neuromuscular degeneration in CMT2D mice
}

\author{
James N. Sleigh (10) ${ }^{1,2}$, Aleksandra M. Mech ${ }^{1}$ and Giampietro Schiavo $\mathbb{B}^{1,2,3}$
}

\begin{abstract}
Dominantly inherited, missense mutations in the widely expressed housekeeping gene, GARS1, cause Charcot-MarieTooth type 2D (CMT2D), a peripheral neuropathy characterised by muscle weakness and wasting in limb extremities. Mice modelling CMT2D display early and selective neuromuscular junction (NMJ) pathology, epitomised by disturbed maturation and neurotransmission, leading to denervation. Indeed, the NMJ disruption has been reported in several different muscles; however, a systematic comparison of neuromuscular synapses from distinct body locations has yet to be performed. We therefore analysed NMJ development and degeneration across five different wholemount muscles to identify key synaptic features contributing to the distinct pattern of neurodegeneration in CMT2D mice. Denervation was found to occur along a distal-to-proximal gradient, providing a cellular explanation for the greater weakness observed in mutant Gars hindlimbs compared with forelimbs. Nonetheless, muscles from similar locations and innervated by axons of equivalent length showed significant differences in neuropathology, suggestive of additional factors impacting on site-specific neuromuscular degeneration. Defective NMJ development preceded and associated with degeneration, but was not linked to a delay of wild-type NMJ maturation processes. Correlation analyses indicate that muscle fibre type nor synaptic architecture explain the differential denervation of CMT2D NMJs, rather it is the extent of post-natal synaptic growth that predisposes to neurodegeneration. Together, this work improves our understanding of the mechanisms driving synaptic vulnerability in CMT2D and hints at pertinent pathogenic pathways.
\end{abstract}

\section{Introduction}

Charcot-Marie-Tooth disease (CMT) is an inherited peripheral neuropathy typified by degeneration of motor and sensory neurons, which triggers progressive muscle wasting and sensory deficits mainly in the feet and hands ${ }^{1}$. When genetic neuropathy results from demyelination and presents with slowed nerve conduction speeds, it is categorised as CMT1, whereas CMT2 results from axon degeneration unrelated to myelin disruption. Mutations in $>100$ genes cause CMT, the majority of which lead to greater dysfunction in lower limbs ${ }^{2}$. There is thus a length-dependency to CMT, such that peripheral nerves

\footnotetext{
Correspondence: James N. Sleigh (j.sleigh@ucl.ac.uk)

${ }^{1}$ Department of Neuromuscular Diseases, UCL Queen Square Institute of

Neurology, University College London, London WC1N 3BG, UK

${ }^{2} U K$ Dementia Research Institute, University College London, London WC1E 6BT, UK

Full list of author information is available at the end of the article

Edited by G. Melino
}

with the longest axons are generally more affected; this suggests that cellular processes most impacted by the extreme morphology of neurons, e.g. axonal transport, may play an important role in pathogenesis ${ }^{3-5}$.

Caused by dominantly inherited, missense mutations in the widely and constitutively expressed gene, GARS1 (ref. ${ }^{6}$ ), the 2D subtype of CMT (CMT2D) usually manifests during adolescence and, unlike most forms of CMT, frequently displays upper limb predominance ${ }^{7,8}$. The GARS1-encoded protein, glycyl-tRNA synthetase (GlyRS), charges glycine to tRNA for protein translation; yet, it is a toxic gain-of-function that likely drives neurodegeneration $^{9-11}$. Contrasting with most other aminoacyl-tRNA synthetases ${ }^{12}$, cytoplasmic and mitochondrial GlyRS isoforms are encoded by a single gene (GARS1) rather than two separate loci ${ }^{13}$. All CMT2D-causing mutations occur downstream of the mitochondrial targeting sequence ${ }^{11}$, while heterozygous mutant Gars mice modelling CMT2D 
show no disturbance in levels of proteins involved in oxidative phosphorylation ${ }^{14}$, together suggesting that mitochondrial dysfunction is unlikely to play a causative role in disease. The mechanisms underlying neuronal selectivity in CMT2D remain unresolved; however, aberrant protein-protein interactions caused by relaxation of the GlyRS structure appear to underlie neomorphic function ${ }^{15,16}$. Indeed, neuropathy-causing mutant GlyRS mis-interacts with the extracellular domains of neuronal transmembrane receptors, neuropilin 1 and tropomyosin receptor kinases (Trks) A- $\mathrm{C}^{16,17}$. Permitting this, GlyRS is secreted from several different cell types and circulates in mammalian serum, likely for a non-canonical and only partially understood function ${ }^{16,18,19}$.

The neuromuscular junction (NMJ) is the specialised synapse connecting lower motor neurons to muscle fibres, and is dysfunctional in several CMT models ${ }^{20-25}$. Indeed, CMT2D mice display loss of NMJ integrity in multiple hindlimb muscles without spinal cord motor neuron degeneration ${ }^{9,26-30}$. By directly comparing a proximal and a distal muscle, we have previously shown that the neuromuscular synapse is an important site of selective and early pathology ${ }^{28}$, replicating the muscle weakness pattern of patients. Also contributing to reduced strength and independently from denervation, these neuropathic mice display pre-synaptic disruption of neurotransmission that correlates with disease severity and worsens with age ${ }^{29}$. Neuromuscular degeneration is replicated in a Drosophila melanogaster model for GARS1 neuropathy and is dependent on toxic accumulation of mutant GlyRS at the $\mathrm{NMJ}^{19}$. Suggestive of a non-cell autonomous mechanism, this pathological build-up requires muscle-secreted, but not neuron-derived, GlyRS, and appears to be mediated by deviant interaction with Plexin $\mathrm{B}^{31}$.

Here, we extend our neuromuscular analyses in CMT2D, through a comprehensive assessment of developmental and degenerative processes at the mouse NMJ across several anatomically and functionally distinct muscles. By correlating varied neuropathic NMJ phenotypes with extensive morphological data on developing and mature wild-type synapses ${ }^{32}$, we have begun to identify key features underlying the selective vulnerability of neuromuscular connections in GARS1 neuropathy.

\section{Materials and methods Animals}

Mouse work was carried out under license from the UK Home Office in accordance with the Animals (Scientific Procedures) Act 1986 and approved by the UCL Queen Square Institute of Neurology Ethical Review Committee. Gars ${ }^{\text {C201R/+ }}$ mice (RRID: MGI 3849420) were maintained as heterozygote breeding pairs on a C57BL/6J background and genotyped as previously described ${ }^{27}$. Animals sacrificed for 1- and 3-month timepoints were 27-35 and
84-95 days old, respectively, and both sexes were used unless otherwise stated.

\section{Grip strength testing}

Grip strength was assessed in forelimbs as previously described ${ }^{17}$.

\section{Muscle dissection and immunohistochemistry}

Muscles were dissected and stained as wholemount preparations as outlined formerly ${ }^{33,34}$. The following antibodies were used to co-stain motor nerve terminals and axons: $1 / 25$ mouse pan anti-synaptic vesicle 2 (SV2, Developmental Studies Hybridoma Bank [DSHB], Iowa City, IA, supernatant); 1/250 mouse anti-neurofilament (2H3, DSHB, supernatant). 1/1000 Alexa Fluor $555 \alpha$ BTX (Thermo Fisher Scientific, Waltham, MA, B35451, RRID:AB_2617152) was used to identify AChRs.

\section{NMJ imaging and analysis}

NMJs were imaged using a LSM780 laser scanning microscope (Zeiss, Oberkochen, Germany). Denervation, polyinnervation, and perforation analyses were performed as detailed elsewhere ${ }^{28,33}$.

\section{Statistical analysis}

Data were assumed to be normally distributed unless evidence to the contrary could be provided by the D'Agostino and Pearson omnibus normality test, while equal variance between groups was assumed. GraphPad Prism 8 (version 8.4.0, La Jolla, CA) was used for all statistical tests, which were all two-sided. Datasets were statistically compared using a one- or two-way analysis of variance (ANOVA) followed by either Sidak's or Tukey's multiple comparisons test. Correlation was assessed using Pearson's product moment correlation. To maintain an $\alpha$ of 0.05 , Bonferroni correction was applied to all correlation analyses when performing multiple tests. Sample sizes, which were pre-determined using previous experience $^{28}$ and power calculations, are reported in all figure legends and represent biological replicates (i.e. individual animals). Once imaged, no muscles were excluded from analyses. As no treatment groups were involved, randomisation was not performed. All reported error bars depict standard error of the mean (SEM). All analyses were performed blinded to genotype.

\section{Results}

NMJ denervation underlies greater hindlimb weakness in CMT2D mice

Mutant Gars mice display a developmental perturbation of sensory neuron fate in hindlimb-innervating lumbar dorsal root ganglia $(\mathrm{DRG})^{17}$, which is not present in forelimb-innervating cervical DRG ${ }^{35}$, suggestive of lengthdependent phenotypes in CMT2D mice. To determine 


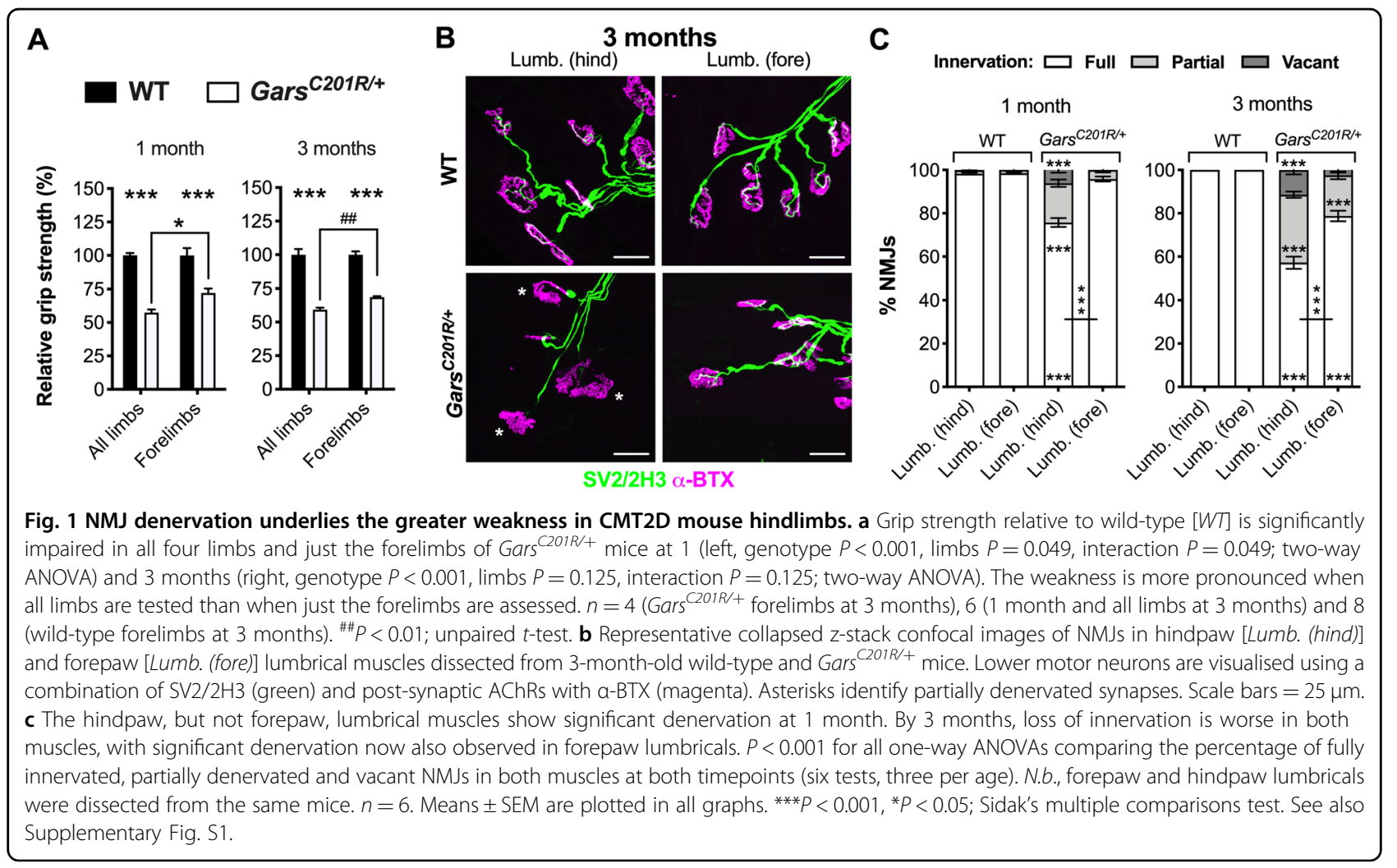

whether the neuromuscular system displays a similar pathological pattern, we performed grip strength testing of mild Gars ${ }^{\mathrm{C} 201 R /+}$ mice $^{27}$. Previous data indicate that muscle function is impaired at both 1 and 3 months in male and female Gars ${ }^{C 201 R /+}$ mice when all limbs are simultaneously tested ${ }^{17}$. Assessing just forelimb muscle function of female wild-type and Gars ${ }^{C 201 R /+}$ mice at the same timepoints, we observed a similar early and persistent deficit in CMT2D strength (Supplementary Fig. S1). Hindlimbs alone cannot be tested because of the manner in which mice grasp the grip strength meter used. Nevertheless, to compare grip strength from all limbs ${ }^{17}$ with forelimb data generated here, we normalised to wildtype at each timepoint in each limb category. The relative forelimb strength was significantly greater at both ages compared with all limbs ( $72 \%$ vs. $57 \%$ and $69 \%$ vs. $59 \%$, Fig. 1a), suggesting that CMT2D mice may display greater weakness in hindlimbs.

Lumbrical muscles involved in hindpaw clasping and grip strength, show a progressive loss of lower motor neuron connectivity in mutant Gars mice, correlating with overall model severity ${ }^{28}$. To compare NMJ innervation between a hindlimb and a forelimb muscle of similar function and relative limb location, we dissected both hindpaw and forepaw lumbrical muscles and stained them with antibodies against SV2/2H3 to visualise motor neurons, and $\alpha$-bungarotoxin $(\alpha-B T X)$ to identify post- synaptic acetylcholine receptors (AChRs) (Fig. 1b). At 1 month, hindlimb lumbricals displayed significantly more partially and fully (i.e. vacant) denervated NMJs than wild-type mice, becoming worse by 3 months (Fig. 1c). In contrast, forelimb lumbricals displayed no significant loss of innervation at 1 month, with comparatively mild denervation manifesting by 3 months. When statistically compared, loss of NMJ integrity was consistently greater in hindpaw lumbricals, indicating that neuromuscular denervation contributes to the greater hindlimb weakness of CMT2D mice.

\section{Impaired NMJ maturation precedes denervation}

Prior to denervation, hindpaw lumbricals in CMT2D mice display impairments in key maturation events, such as synapse elimination and post-synaptic plaque-topretzel migration ${ }^{28}$; however, these developmental delays were not seen in a postural, anterior abdominal muscle called the transversus abdominis (TVA) ${ }^{36}$, which also lacked denervation at 3 months in mutant Gars mice ${ }^{28}$. To test whether this link between perturbed NMJ maturation and degeneration is consistent across CMT2D muscles, we assessed synaptic development in hindpaw and forepaw lumbricals (Fig. 2). At birth, post-synaptic endplates are contacted by several different motor neurons (see Fig. 2a for an example) and, through a process called synapse elimination, go from being polyinnervated 

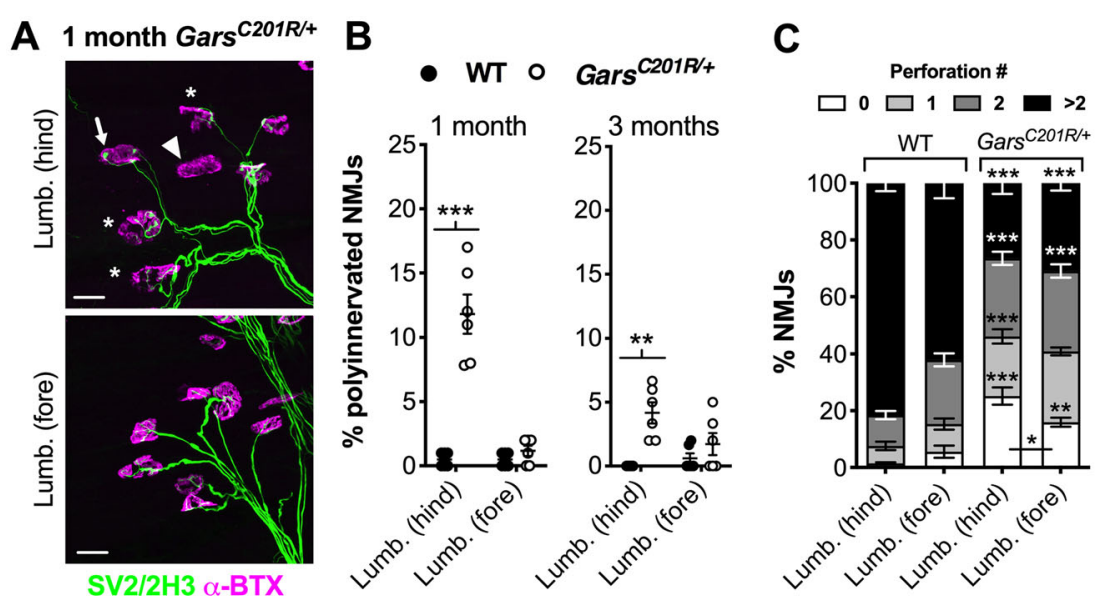

Fig. 2 Impaired CMT2D NMJ maturation is linked to denervation. a Representative collapsed z-stack confocal images of NMJs in hindpaw [Lumb. (hind)] and forepaw [Lumb. (fore)] lumbrical muscles dissected from 1-month-old Gars ${ }^{\text {C201R/+ }}$ mice. Lower motor neurons are visualised using a combination of SV2/2H3 (green) and post-synaptic AChRs with a-BTX (magenta). Asterisks identify partially denervated synapses, the arrowhead a vacant synapse, and the arrow a polyinnervated NMJ. Scale bars $=25 \mu \mathrm{m}$. b Hindpaw, but not forepaw, lumbrical muscles of Gars ${ }^{C 201 R /+}$ mice display significantly more polyinnervated NMJs at 1 month compared with wild-type (left, genotype $P<0.001$, muscle $P<0.001$, interaction $P<0.001$; twoway repeated measures ANOVA). This pattern is also present at 3 months (right, genotype $P=0.001$, muscle $P=0.191$, interaction $P=0.029$; two-way repeated measures ANOVA), although there are fewer polyinnervated NMJs in the hindpaw lumbricals at this stage, indicative of a delay rather than cessation of synapse elimination. $\mathbf{c}$ Both hindpaw and forepaw lumbrical NMJs show post-synaptic maturation deficiency as assessed by counting the perforations per endplate. This defect was significantly worse in hindpaw lumbricals. $P<0.001$ for all one-way ANOVAs comparing the percentage of NMJs with none, one, two, and more than two perforations in both muscles (i.e. four tests). N.b., hindpaw and forepaw muscles are the same as those analysed in Fig. 1. $n=6$. Means \pm SEM are plotted in all graphs. ${ }^{* *} P<0.001,{ }^{* *} P<0.01,{ }^{*} P<0.05$; Sidak's multiple comparisons test.

to monoinnervated by 2 weeks in mice ${ }^{37,38}$. In tandem, AChRs migrate and become concentrated in close apposition to motor nerve terminals, resulting in their conversion from simple, circular plaques at birth to complex pretzel-like structures with increasing numbers of perforations ${ }^{39}$. Delayed synapse elimination was replicated in Gars ${ }^{C 201 R /+}$ hindpaw lumbrical NMJs, which showed significant polyinnervation at 1 month and 3 months (Fig. 2b). Contrastingly, forepaw lumbricals showed no such defect. To assess post-synaptic development, perforations per endplate were counted, with higher numbers indicative of advanced maturation. Once again, hindpaw lumbrical NMJs showed impaired AChR development, which was also present in forepaw lumbricals, albeit not to the same extent (Fig. 2c). These data indicate that defective pre-synaptic maturation is associated with subsequent neuromuscular degeneration, whereas postsynaptic disruption may represent a systemic effect, as it is also seen in the non-denervated $\mathrm{TVA}^{28}$.

\section{Hindlimb FDB muscles also display delayed NMJ development and degeneration}

To extend our analyses into additional hindlimb and forelimb muscles, we stained NMJs of hindpaw flexor digitorum brevis (FDB) and forelimb epitrochleoanconeus (ETA) muscles dissected from 3-month-old mice (Fig. 3a). FDB muscles aid hindpaw opening and the ETA contributes to forearm supination ${ }^{40,41}$, and both can be wholemount stained to assess innervation ${ }^{32}$. The FDB displayed defective synapse elimination associated with severe denervation, whereas the ETA was unaffected (Fig. $3 \mathrm{~b}, \mathrm{c})$. These data uphold that CMT2D hindlimbs display greater pathology, and that impaired NMJ development consistently accompanies degeneration.

\section{CMT2D polyinnervation correlates with degeneration and is independent of synapse elimination}

Including previous data from the TVA ${ }^{28}$, we have now assessed NMJ polyinnervation and denervation at 3 months in five wholemount muscles (Fig. 4a). We detected a broad spectrum of denervation (partial and full combined) in CMT2D mice, ranging from $2 \%$ in the TVA to $63 \%$ in the FDB (Fig. 4b, Supplementary Table S1). A similar phenotypic continuum was identified for disturbed synapse elimination, with $0.7 \%$ of TVA NMJs remaining polyinnervated and $7.3 \%$ of FDB NMJs (Fig. 4c, Supplementary Table S1). We tested the relationship between these two phenotypes and found a highly significant positive correlation between percentage of vacant and polyinnervated synapses occurring at 3 months (Fig. 4d). A similar correlation was present when vacant and partially denervated synapses were combined (\% denervation, Fig. 4e). To determine whether perturbed synapse elimination is simply a delay of the physiological process 

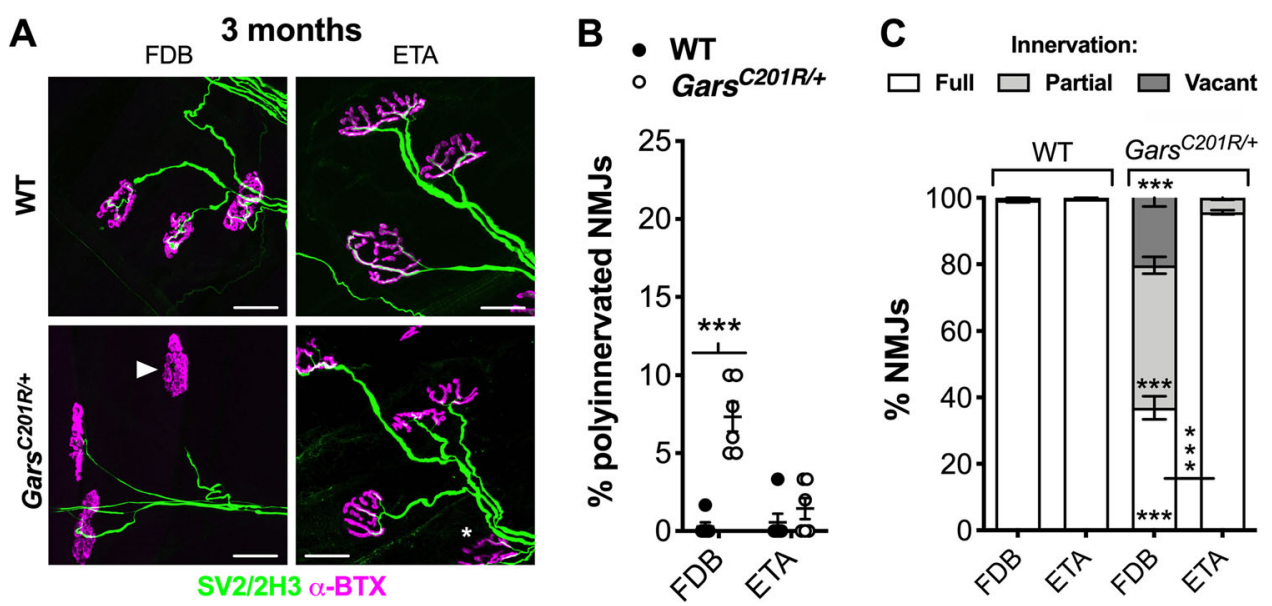

Fig. 3 Hindlimb muscles behave similarly and show marked differences with forelimb muscles. a Representative collapsed z-stack confocal images of NMJs in FDB and ETA muscles dissected from 3-month-old wild-type and Gars ${ }^{\mathrm{C} 201 R /+}$ mice. Lower motor neurons are visualised using a combination of SV2/2H3 (green) and post-synaptic AChRs with a-BTX (magenta). The asterisk identifies a partially denervated NMJ and the arrowhead a fully denervated synapse. Scale bars $=25 \mu \mathrm{m}$. b The FDB, but not ETA, muscle of Gars ${ }^{\mathrm{C} 201 R /+}$ mice displays significantly more polyinnervated NMJs at 3 months (genotype $P<0.001$, muscle $P=0.005$, interaction $P<0.001$; two-way repeated measures ANOVA). c At 3 months, denervation in the FDB muscle is severe, whereas the ETA shows little degeneration. $P<0.001$ for all one-way ANOVAs comparing the percentage of fully innervated, partially denervated and vacant NMJs in both muscles (i.e. three tests). $n=6$. Means \pm SEM are plotted in all graphs. ${ }^{* * *} P<0.001$; Sidak's multiple comparisons test.

occurring in wild-type animals, we correlated the CMT2D motor input counts with previously generated ${ }^{32}$ P7 wild-type polyinnervation data from the five muscles (Fig. 4f). No correlation was identified, suggesting that impaired synapse elimination in mutant Gars mice may be caused by selective, muscle-specific perturbation of pathways relevant to removal of supernumerary motor axons, as opposed to a systemic slow-down in the process.

\section{Fibre type does not associate with CMT2D denervation}

Motor neurons innervating hindpaw muscles (FDB and lumbricals) are more impacted in CMT2D mice than those targeting forepaws (lumbricals) followed by more proximal muscles (ETA and TVA) (Fig. 4). This indicates a general distal-to-proximal vulnerability axis consistent with length-dependent axon degeneration. However, adjacent muscles innervated by neurons of similar length show significant distinctions in denervation (Supplementary Table S1), suggesting that additional factors contribute to NMJ vulnerability. Fast-fatiguable motor neurons innervating fast twitch muscle fibres are more susceptible to degeneration in amyotrophic lateral sclerosis (ALS) than motor nerves contacting slow twitch fibres $^{42}$. To assess whether this is also observed in CMT2D, we correlated previously published fast twitch fibre type percentages ${ }^{32,40,41,43-45}$ with mutant Gars mouse denervation (Supplementary Fig. S2). No relationship was observed indicating that fibre type is unlikely to be driving inter-muscle disparities in neuropathy.

\section{CMT2D denervation correlates with demand for post-natal NMJ growth}

We then evaluated NMJ structure as a possible determinant in neuropathology, since NMJs with smaller synaptic volumes have less neuronal input to lose to become fully denervated. Using a robust and standardised semi-automated workflow called NMJ-morph ${ }^{46}$, we have previously generated detailed data on morphology of developing (P7) and mature (P31-32) wild-type NMJs in the same five muscles assessed here ${ }^{32}$. We found that developing synapses show greater inter-muscle variability and that post-natal NMJ growth occurs at different rates across muscles ${ }^{32}$-two phenotypes that may provide a substrate for differential NMJ degeneration in CMT2D. We therefore first correlated all 41 morphological variables from P7 and P31-32 wild-type NMJs with the percentage of vacant synapses in 3-month mutant Gars mice (Supplementary Table S2); however, no significant relationships were identified. We then tested for correlations between morphology and combined percentages of partially and fully denervated NMJs (Supplementary Table S3). From 41 variables, only AChR perimeter of developing NMJs showed a significant interaction, indicating that a smaller AChR perimeter associates with greater denervation levels (Supplementary Fig. S3). Given that many of the assessed NMJ variables are interrelated (e.g. AChR perimeter/area), the finding that only one out of 82 tests showed a significant correlation suggests that synaptic architecture has little impact on neuropathy. 

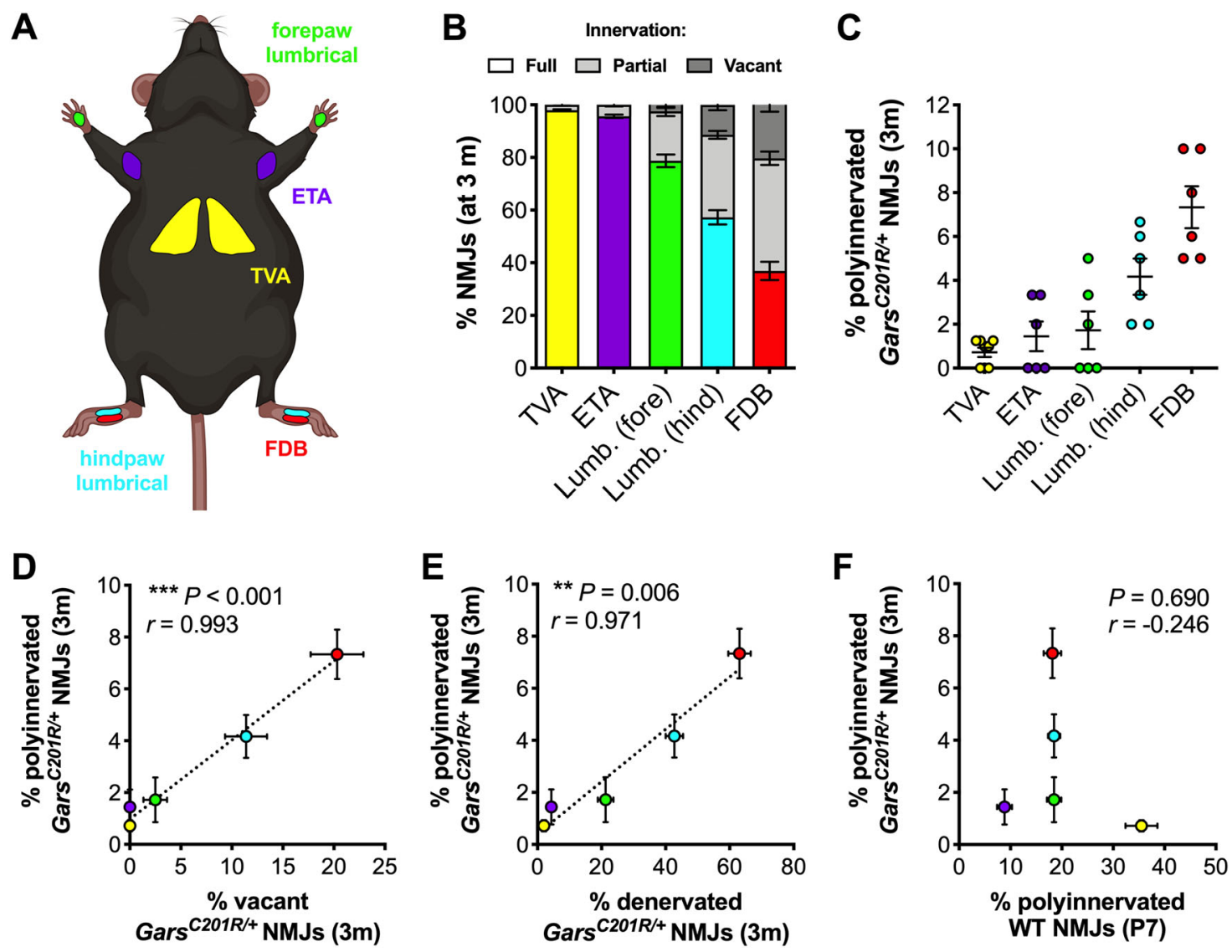

Fig. 4 CMT2D NMJ polyinnervation correlates with denervation and is not simply a delay of wild-type synapse elimination. a Five thin and flat muscles were analysed in this study: transversus abdominis (TVA, yellow), epitrochleoanconeus (ETA, purple), forelimb lumbricals (green, Lumb. [fore]), hindlimb lumbricals (cyan, Lumb. [hind]) and flexor digitorum brevis (FDB, red). The schematic was created with BioRender (https://biorender. com). $\mathbf{b}$ The five muscles display a spectrum of vulnerability to denervation in Gars ${ }^{201 R /+}$ mice at 3 months; the TVA remains unaffected, while the FDB muscles show stark degeneration. c Defective synapse elimination at 3 months is more pronounced in muscles with greater denervation. $\mathbf{d}$, e There is a significant correlation between the percentage of polyinnervation observed in each muscle and the percentage of vacant $\left(\mathbf{d},{ }^{* * *} P<0.001\right.$, $r=0.993)$ or denervated $\left(\mathbf{e}^{*}{ }^{*} P=0.006, r=0.971\right) \mathrm{NMJs}$ at 3 months in Gars ${ }^{\mathrm{C} 201 R /+}$ mice. $\mathbf{f}$ There is no correlation between the percentage of wildtype NMJ polyinnervation at P7 and the polyinnervation percentage at 3 months in Gars ${ }^{\mathrm{C} 201 R /+}$ mice $(P=0.690, r=-0.246)$, suggesting that the mutant phenotype is not due to a delay of wild-type synapse elimination. See Supplementary Table S1 for pairwise statistical testing between muscles in panels (b) and (c). Data in panels (e) and (f) were analysed by Pearson's product moment correlation. The colour-coding of muscles in panels $(\mathbf{e})$ and $(\mathbf{f})$ is maintained from panels $(\mathbf{a}-\mathbf{c})$. TVA data ${ }^{28}$ and wild-type P7 polyinnervation data ${ }^{32}$ were generated for previous studies. $n=6$ (all muscles except TVA) and 8 (TVA). Means \pm SEM are plotted in all graphs.

Finally, we assessed the role of post-natal NMJ growth and development by correlating the percentage change in each morphological variable with the extent of mutant Gars degeneration. Several different pre-synaptic (terminal area, number of branches and branch length), post-synaptic (AChR area) and overlapping (synaptic contact area) variables significantly correlated with the percentage of vacant CMT2D NMJs (Fig. 5a, Supplementary Table S4). Two further presynaptic variables (branch point number and complexity) significantly associated with the percentage of denervated (partial and full) mutant NMJs (Fig. 5b, Supplementary Table S5). All seven correlations were positive, indicating that NMJs undergoing greater post-natal change in morphology are more prone to degeneration in CMT2D mice.

\section{Discussion}

CMT patients usually present with weakness and atrophy in the feet and then hands, indicating that peripheral neurons with longer axons are more impacted by the disease. However, mutations in a few genes, including GARS1, can cause an upper limb predominance ${ }^{7,8,47}$. This suggests that while axon length is important, additional characteristics contribute to pathogenesis and the spectrum of motor neuron involvement. Identification of such factors may elucidate pathomechanisms and provide potential targetable pathways. While we did not observe upper limb weakness in mutant Gars mice (possible reasons for which are discussed elsewhere ${ }^{35}$ ), we do observe significant distinctions in NMJ pathology between 


\section{A i pre-synaptic}
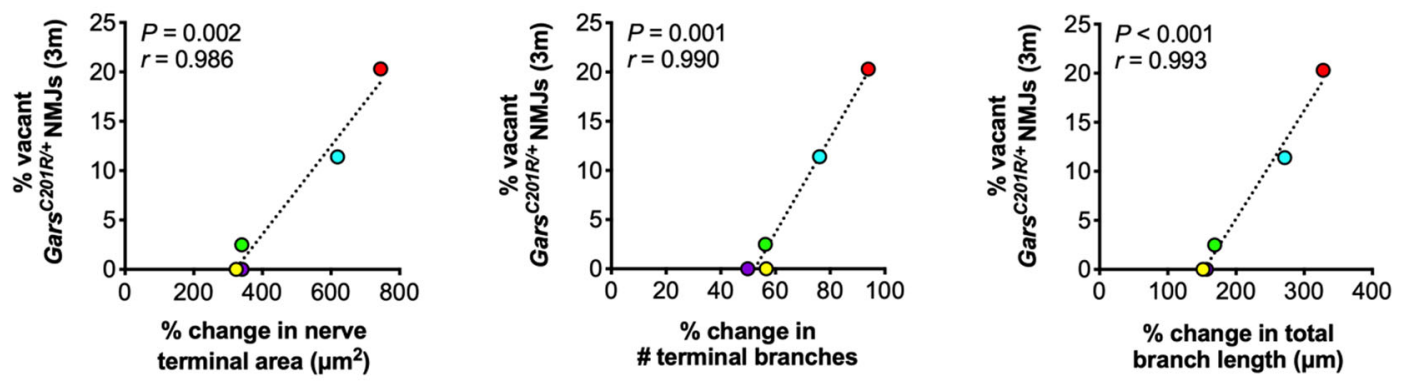

\section{ii post-synaptic}

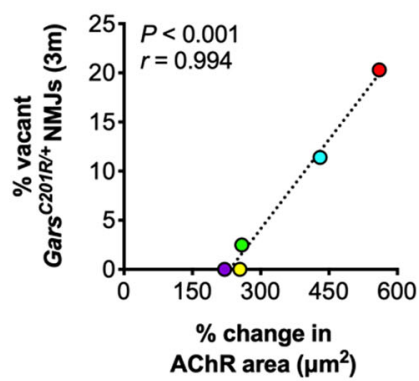

\section{iii overlapping}

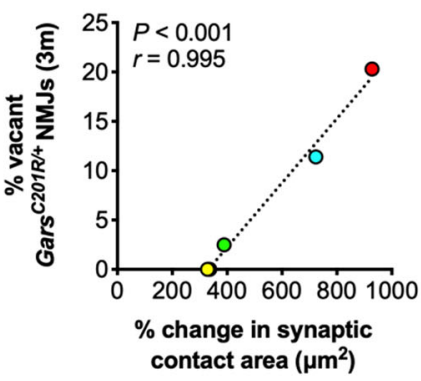

\section{B pre-synaptic}
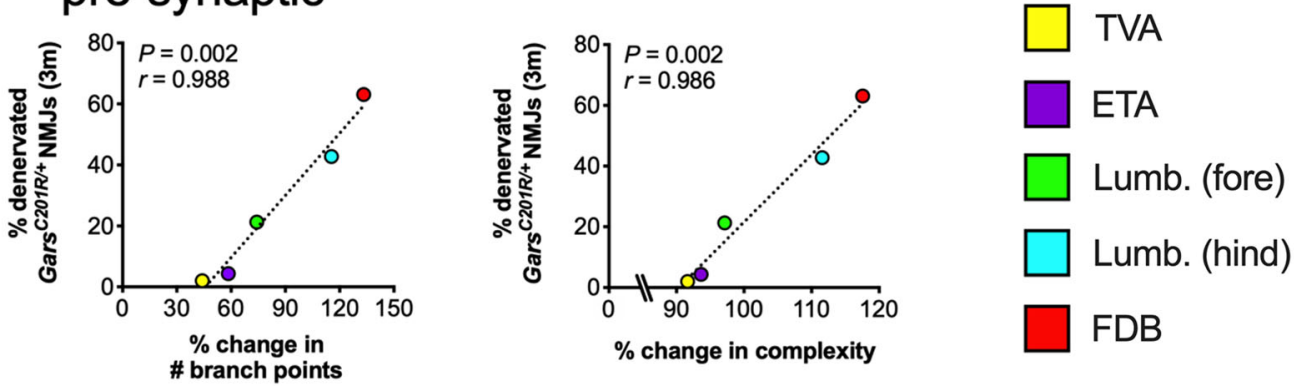

Fig. 5 NMJs that undergo greater post-natal morphological change are more vulnerable to degeneration in CMT2D mice. The percentages of vacant (a) and denervated (b) NMJs in 3-month Gars ${ }^{\mathrm{C} 201 R /+}$ mice were correlated with post-natal NMJ growth in 20 different pre-synaptic (i), postsynaptic (ii) and overlapping (iii) morphological variables. Wild-type P7 to P31-32 morphological data ${ }^{32}$ and TVA vacancy data ${ }^{28}$ were previously published. Correlation was assessed by calculating Pearson's product moment correlation coefficient $(r)$, the results of which are presented in Supplementary Table S4 and Supplementary Table S5 along with associated $P$ values. Only significant correlations are presented, i.e. Bonferronicorrected $P<0.00256 . n=6$ (all muscles except TVA) and 8 (TVA). Lumb. (fore), forepaw lumbricals; Lumb. (hind), hindpaw lumbricals.

adjacent muscles innervated by motor neurons of similar length. This suggests that, like in human patients, CMT2D mouse motor neurons possess features that modify their predisposition to neuropathology.

We first assessed the impact of muscle fibre type on neuropathy, because large, fast-fatiguable motor neurons targeting fast twitch fibres are more vulnerable in $\mathrm{ALS}^{42}$, a motor neuron disease with parallels to CMT. There was no correlation between fibre type and CMT2D degeneration, suggesting that in contrast to ALS, muscle fibre types have little impact on motor neuropathy. Perhaps impacting accuracy, fibre type percentages, including from rat TVA, were obtained from several studies; thus, in the future fibre typing should be performed in parallel across all five muscles.

Since pathology in mutant Gars mice and patients is restricted to distal segments of peripheral nerves, we then probed NMJ morphology. Again, we found little evidence to indicate that distinctions in pre- or post-synaptic architecture cause the spectrum of synapse loss. However, we did find that CMT2D denervation correlated closely with wild-type NMJ change and development. This suggests that motor terminals under the greatest pressures to grow and mature during early post-natal life, perhaps due to different functions of their associated muscles ${ }^{32}$, are more likely to degenerate. Possibly contributing to this, 
severe CMT2D mice possess fewer mitochondria in presynaptic motor terminals in the proximal levator auris longus muscle ${ }^{29}$. Accordingly, motor neurons with higher bioenergetic capacity and thus energy supply are more resistant to disease in spinal muscular atrophy mice ${ }^{48}$, while computational modelling indicates energetic demand also contributes to selective vulnerability in ALS $^{49}$.

While developmental demand may contribute to early synaptic disruption, GARS1-associated neuropathy is progressive and denervation continues in CMT2D mice up to 3 months and beyond. Additional mechanisms are therefore likely driving the life-long neurodegeneration caused by mutant GlyRS.

Synapse elimination is mediated by reciprocal nervemuscle signalling and electrical activity to refine nervous system architecture ${ }^{38,50}$. The strong correlation across CMT2D muscles between polyinnervated and denervated synapses, and the lack of association with wild-type polyinnervation, indicate that synapse elimination is linked to pathology and not caused by systemic interruption.

Synapse elimination delay is unlikely to be driving denervation for two reasons; firstly, having multiple motor neurons innervating an endplate is probably beneficial from an innervation standpoint as several motor nerve terminals must degenerate for full denervation to occur. Corroborating this, the percentage of denervation (partial and full) across all FDB NMJs is $63 \pm 4 \%$ at 3 months, whereas at polyinnervated synapses, only $6 \pm 4 \%$ of NMJs show signs of degeneration (data not shown). Secondly, mechanisms mediating removal of supernumerary motor inputs share many features with neurodegenerative pathways $^{51}$, and hence a delay in elimination would likely also postpone neuropathy. Alternatively, the phenotype could represent a compensatory response to degeneration, similar in essence to Schwann cell-mediated neuronal sprouting ${ }^{52}$ or pre-synaptic homeostatic plasticity ${ }^{53}$. However, if this were the case, it would probably be observed throughout the lifespan of CMT2D mice and in different neuromuscular disease models, but this does not appear to occur. We therefore suggest that the polyinnervation phenotype is an epiphenomenon resulting from aberrant interaction of mutant GlyRS with one or several key trans-synaptic proteins. There is precedent for this in a CMT2D Drosophila model; muscle-secreted mutant GlyRS aberrantly accumulates at NMJs prior to degeneration ${ }^{19}$ and coincides with neurodevelopmental wiring defects ${ }^{31}$. This phenocopies ectopic motor neuron branching caused by loss-of-function mutations in plex $A$ and plexB, which encode neuronal transmembrane proteins that bind secreted semaphorins to facilitate axonal guidance, retrograde signalling and synaptic plasticity ${ }^{54,55}$. This observation led to the finding that plexin $B$ serves as an erroneous binding partner for mutant GlyRS at the NMJ; however, while mutant GlyRS interferes with the fidelity of axon guidance pathways causing wiring defects, this was de-coupled from NMJ pathologies, i.e. was an epiphenomenon $^{31}$.

We have previously shown that mutant GlyRS aberrantly interacts with neurotrophin receptors (TrkA-C) ${ }^{17}$, of which TrkB is the main one present at the mammalian $\mathrm{NMJ}^{56,57}$. TrkB and its ligand, brain-derived neurotrophic factor (BDNF), play an important role in NMJ synapse elimination ${ }^{57-59}$. This may, in part, be driven by BDNFmediated clustering in motor nerve terminals of voltagegated calcium channels, which facilitate calcium influx, synaptic neurotransmission, and NMJ maturation ${ }^{60-63}$. Mutant GlyRS mis-interacting with TrkB at the NMJ could delay synapse elimination through impairing calcium channel insertion into pre-synaptic motor terminals, thereby hindering neurotransmission and neuronal activity. Consistent with this idea, it has been shown that CMT2D mice display pre-synaptic transmission defects, including reduced frequency of spontaneous neurotransmitter release, which is calcium transientdependent ${ }^{29}$. A primary mechanism causing the weakened synaptic transmission was ultimately not identified, instead it was suggested that several different release processes function sub-optimally ${ }^{29}$. It is therefore conceivable that accumulation of mutant GlyRS at NMJs through association with TrkB, akin to the synaptic buildup of pathological protein observed in the fly model ${ }^{19,31}$, may be contributing to the impaired synaptic function prior to NMJ degeneration.

Upon BDNF binding, TrkB is internalised into neuron terminals. Activated receptor complexes can then precipitate local signalling events such as those just discussed or become sorted into signalling endosomes for longrange retrograde axonal transport critical to pro-survival gene transcription ${ }^{64,65}$. The erroneous association of mutant GlyRS with TrkB at motor nerve terminals may thus diminish retrograde neurotrophic signalling, contributing to neuropathy. Correspondingly, signalling endosome transport is impaired in primary sensory neurons cultured from severe mutant Gars mice ${ }^{66}$, while mutations in essential signalling endosome retrograde effectors, Rab7 (ref. ${ }^{67}$ ) and dynactin, cause peripheral neuropathy ${ }^{68,69}$. In addition, activation of IGF1R, a neurotrophic factor receptor found at the NMJ, is involved in regulating signalling endosome dynamics in motor neurons $^{70}$, which may be a common function of similar receptors like $\operatorname{TrkB}^{71}$. Furthermore, high levels of phosphorylated IGF1R at NMJs may confer resistance to motor degeneration in ALS mice ${ }^{72}$, suggesting that levels of neurotrophic factors and/or their receptors (e.g. BDNF/ TrkB) could indeed play a role in selective vulnerability to motor terminal loss in peripheral neuropathy. 
Alternatively, mutant GlyRS sequestration of TrkB could divert BDNF to bind to the pan-neurotrophin death receptor, $\mathrm{p} 75^{\mathrm{NTR}}$, resulting in over-activation of degenerative signals ${ }^{73}$. Consistent with these views and the importance of TrkB signalling to peripheral synapse integrity, reduced TrkB activity is known to impair NMJ neurotransmission, maturation and innervation, without impacting motor neuron number ${ }^{56,74}$, phenotypes very similar to those found in CMT2D mice. It is therefore conceivable that different levels of TrkB and/or BDNF at NMJs across muscles contribute to both the differential denervation and subverted synaptic maturation.

In summary, by assessing developmental and degenerative NMJ phenotypes across five different wholemount muscles from CMT2D mice, we have identified a spectrum of vulnerability to neurodegeneration, which is fully supported by findings in two muscles from the more severe mutant Gars mouse ${ }^{28}$. Through correlation analyses with muscle fibre type, NMJ architecture, and postnatal synaptic growth, we identified that the magnitude of developmental demand likely contributes to neuromuscular synapse loss in mutant Gars mice. We believe that these results point the way forward for an improved understanding of the molecular mechanisms driving differences in synaptic vulnerability to neuropathy.

\section{Acknowledgements}

The authors would like to thank Stuart J. Grice (University of Oxford) and David Villarroel-Campos (University College London) for critical comments on the manuscript and Anna-Leigh Brown (University College London) for fruitful discussions. This work was supported by the Medical Research Council Career Development Award (MR/S006990/1) [J.N.S.], the Wellcome Trust Sir Henry Wellcome Postdoctoral Fellowship (103191/Z/13/Z) [J.N.S.], the Wellcome Trust Senior Investigator Award (107116/Z/15/Z) [G.S.], the European Union's Horizon 2020 Research and Innovation programme under grant agreement 739572 [G.S.], and the UK Dementia Research Institute Foundation award (UKDRI-1005) [G.S.].

\section{Author details \\ 'Department of Neuromuscular Diseases, UCL Queen Square Institute of Neurology, University College London, London WC1N 3BG, UK. ${ }^{2}$ UK Dementia Research Institute, University College London, London WC1E 6BT, UK. ${ }^{3}$ Discoveries Centre for Regenerative and Precision Medicine, University College London Campus, London WC1N 3BG, UK}

\section{Author contributions}

J.N.S. conceived the experiments; J.N.S. and A.M.M. performed the research; J.N. S. and A.M.M. analysed the data; G.S. provided expertise and discussion; J.N.S. wrote the paper with input from all authors. All authors approved submission of this work.

\section{Data availability}

The data that support the findings of this study are available from the corresponding author upon reasonable request.

\section{Conflict of interest}

The authors declare that they have no conflict of interest.

\section{Publisher's note}

Springer Nature remains neutral with regard to jurisdictional claims in published maps and institutional affiliations.
Supplementary Information accompanies this paper at (https://doi.org/ 10.1038/s41419-020-02798-y).

Received: 21 May 2020 Revised: 10 July 2020 Accepted: 13 July 2020 Published online: 23 July 2020

\section{References}

1. Reilly, M. M., Murphy, S. M. \& Laurá, M. Charcot-Marie-Tooth disease. J. Peripher. Nerv. Syst. 16, 1-14 (2011).

2. Pipis, M., Rossor, A. M., Laura, M. \& Reilly, M. M. Next-generation sequencing in Charcot-Marie-Tooth disease: opportunities and challenges. Nat. Rev. Neurol. 15, 644-656 (2019)

3. Prior, R., van Helleputte, L., Benoy, V. \& van den Bosch, L. Defective axonal transport: a common pathological mechanism in inherited and acquired peripheral neuropathies. Neurobiol. Dis. 105, 300-320 (2017).

4. Beijer, D., Sisto, A., van Lent, J., Baets, J. \& Timmerman, V. Defects in axonal transport in inherited neuropathies. J. Neuromuscul. Dis. 6, 401-419 (2019).

5. Sleigh, J. N., Rossor, A. M., Fellows, A. D., Tosolini, A. P. \& Schiavo, G. Axonal transport and neurological disease. Nat. Rev. Neurol. 15, 691-703 (2019).

6. Antonellis, A. et al. Glycyl tRNA synthetase mutations in Charcot-Marie-Tooth disease type 2D and distal spinal muscular atrophy type V. Am. J. Hum. Genet 72, 1293-1299 (2003).

7. Sivakumar, K. et al. Phenotypic spectrum of disorders associated with glycyltRNA synthetase mutations. Brain 128, 2304-2314 (2005).

8. Antonellis, A., Goldfarb, L. G. \& Sivakumar, K. in GeneReviews ${ }^{\circledR}$ (eds. Adam, M. P. et al.) (University of Washington, Seattle, 2018).

9. Motley, W. W. et al. Charcot-Marie-Tooth-linked mutant GARS is toxic to peripheral neurons independent of wild-type GARS levels. PLoS Genet. 7, e1002399 (2011).

10. Boczonadi, V., Jennings, M. J. \& Horvath, R. The role of tRNA synthetases in neurological and neuromuscular disorders. FEBS Lett. 592, 703-717 (2018).

11. Wei, N., Zhang, Q. \& Yang, X.-L. Neurodegenerative Charcot-Marie-Tooth disease as a case study to decipher novel functions of aminoacyl-tRNA synthetases. J. Biol. Chem. 294, 5321-5339 (2019).

12. Antonellis, A. \& Green, E. D. The role of aminoacyl-tRNA synthetases in genetic diseases. Annu. Rev. Genomics Hum. Genet. 9, 87-107 (2008).

13. Alexandrova, J., Paulus, C., Rudinger-Thirion, J., Jossinet, F. \& Frugier, M. Elaborate UORF/IRES features control expression and localization of human glycyl-tRNA synthetase. RNA Biol. 12, 1301-1313 (2015).

14. Boczonadi, V. et al. Mutations in glycyl-tRNA synthetase impair mitochondrial metabolism in neurons. Hum. Mol. Genet. 27, 2187-2204 (2018).

15. He, W. et al. Dispersed disease-causing neomorphic mutations on a single protein promote the same localized conformational opening. Proc. Natl Acad. Sci. USA 108, 12307-12312 (2011).

16. He, W. et al. CMT2D neuropathy is linked to the neomorphic binding activity of glycyl-tRNA synthetase. Nature 526, 710-714 (2015).

17. Sleigh, J. N. et al. Trk receptor signaling and sensory neuron fate are perturbed in human neuropathy caused by Gars mutations. Proc. Natl Acad. Sci. USA 114, E3324-E3333 (2017).

18. Park, M. C. et al. Secreted human glycyl-tRNA synthetase implicated in defense against ERK-activated tumorigenesis. Proc. Natl Acad. Sci. USA 109, E640-E647 (2012).

19. Grice, S. J. et al. Dominant, toxic gain-of-function mutations in gars lead to non-cell autonomous neuropathology. Hum. Mol. Genet. 24, 4397-4406 (2015).

20. Court, F. A., Brophy, P. J. \& Ribchester, R. R. Remodeling of motor nerve terminals in demyelinating axons of periaxin-null mice. Glia 56, 471-479 (2008).

21. Ang, E.-T. et al. Motor axonal sprouting and neuromuscular junction loss in an animal model of Charcot-Marie-Tooth disease. J. Neuropathol. Exp. Neurol. 69, 281-293 (2010)

22. Scurry, A. N. et al. Structural and functional abnormalities of the neuromuscular junction in the Trembler-J homozygote mouse model of congenital hypomyelinating neuropathy. J. Neuropathol. Exp. Neurol. 75, 334-346 (2016).

23. Sabblah, T. T. et al. A novel mouse model carrying a human cytoplasmic dynein mutation shows motor behavior deficits consistent with CharcotMarie-Tooth type 20 disease. Sci. Rep. 8, 1739 (2018).

24. Cipriani, S. et al. Neuromuscular junction changes in a mouse model of Charcot-Marie-Tooth disease type 4C. Int. J. Mol. Sci. 19, 4072 (2018). 
25. Soh, M. S. et al. Disruption of genes associated with Charcot-Marie-Tooth type 2 lead to common behavioural, cellular and molecular defects in Caenorhabditis elegans. PLOS ONE 15, e0231600 (2020).

26. Seburn, K. L., Nangle, L. A., Cox, G. A., Schimmel, P. \& Burgess, R. W. An active dominant mutation of glycyl-tRNA synthetase causes neuropathy in a Charcot-Marie-Tooth 2D mouse model. Neuron 51, 715-726 (2006).

27. Achilli, F. et al. An ENU-induced mutation in mouse glycyl-tRNA synthetase (GARS) causes peripheral sensory and motor phenotypes creating a model of Charcot-Marie-Tooth type 2D peripheral neuropathy. Dis. Model. Mech. 2, 359-373 (2009).

28. Sleigh, J. N., Grice, S. J., Burgess, R. W., Talbot, K. \& Cader, M. Z. Neuromuscular junction maturation defects precede impaired lower motor neuron connectivity in Charcot-Marie-Tooth type 2D mice. Hum. Mol. Genet. 23 2639-2650 (2014).

29. Spaulding, E. L. et al. Synaptic deficits at neuromuscular junctions in two mouse models of Charcot-Marie-Tooth type 2d. J. Neurosci. 36, 3254-3267 (2016).

30. Morelli, K. H. et al. Allele-specific RNA interference prevents neuropathy in Charcot-Marie-Tooth disease type 2D mouse models. J. Clin. Invest. 129, 5568-5583 (2019).

31. Grice, S. J., Sleigh, J. N. \& Cader, M. Z. Plexin-semaphorin signaling modifies neuromuscular defects in a Drosophila model of peripheral neuropathy. Front. Mol. Neurosci. 11, 55 (2018).

32. Mech, A. M., Brown, A. L., Schiavo, G. \& Sleigh, J. N. Morphological variability is greater at developing than mature mouse neuromuscular junctions. J. Anat. https://doi.org/10.1111/joa.13228 (2020).

33. Sleigh, J. N., Burgess, R. W., Gillingwater, T. H. \& Cader, M. Z. Morphological analysis of neuromuscular junction development and degeneration in rodent lumbrical muscles. J. Neurosci. Methods 227, 159-165 (2014).

34. Sleigh, J. N. et al. Neuropilin 1 sequestration by neuropathogenic mutant glycyl-tRNA synthetase is permissive to vascular homeostasis. Sci. Rep. 7, 9216 (2017).

35. Sleigh, J. N., Mech, A. M., Aktar, T., Zhang, Y. \& Schiavo, G. Altered sensory neuron development in CMT2D mice is site-specific and linked to increased GlyRS levels. Front. Cell. Neurosci. https://doi.org/10.3389/fncel.2020.00232 (2020).

36. Murray, L., Gillingwater, T. H. \& Kothary, R. Dissection of the transversus abdominis muscle for whole-mount neuromuscular junction analysis. J. Vis. Exp. e51162 https://doi.org/10.3791/51162 (2014).

37. Sanes, J. R. \& Lichtman, J. W. Development of the vertebrate neuromuscular junction. Annu. Rev. Neurosci. 22, 389-442 (1999).

38. Bloch-Gallego, E. Mechanisms controlling neuromuscular junction stability. Cell Mol. Life Sci. 72, 1029-1043 (2015).

39. Marques, M. J., Conchello, J. A. \& Lichtman, J. W. From plaque to pretzel: fold formation and acetylcholine receptor loss at the developing neuromuscular junction. J. Neurosci. 20, 3663-3675 (2000).

40. Bradley, S. A., Lyons, P. R. \& Slater, C. R. The epitrochleoanconeus muscle (ETA) of the mouse: a useful muscle for the study of motor innervation in vitro. J. Physiol. 415, 3 (1989).

41. Tarpey, M. D. et al. Characterization and utilization of the flexor digitorum brevis for assessing skeletal muscle function. Skelet. Muscle 8, 14 (2018).

42. Nijssen, J., Comley, L. H. \& Hedlund, E. Motor neuron vulnerability and resistance in amyotrophic lateral sclerosis. Acta Neuropathol. 133, 863-885 (2017).

43. Delp, M. D. \& Duan, C. Composition and size of type I, IIA, IID/X, and IIB fibers and citrate synthase activity of rat muscle. J. Appl. Physiol. 80, 261-270 (1996).

44. Smith, I. C. et al. Potentiation in mouse lumbrical muscle without myosin light chain phosphorylation: is resting calcium responsible? J. Gen. Physiol. 141, 297-308 (2013).

45. Russell, K. A., Ng, R., Faulkner, J. A., Claflin, D. R. \& Mendias, C. L. Mouse forepaw lumbrical muscles are resistant to age-related declines in force production. Exp. Gerontol. 65, 42-45 (2015).

46. Jones, R. A. et al. NMJ-morph reveals principal components of synaptic morphology influencing structure-function relationships at the neuromuscular junction. Open Biol. 6, 160240 (2016).

47. Werheid, F. et al. Underestimated associated features in CMT neuropathies: clinical indicators for the causative gene? Brain Behav. 6, e00451 (2016).

48. Boyd, P. J. et al. Bioenergetic status modulates motor neuron vulnerability and pathogenesis in a zebrafish model of spinal muscular atrophy. PLoS Genet. 13, e1006744 (2017).
49. Le Masson, G., Przedborski, S. \& Abbott, L. F. A computational model of motor neuron degeneration. Neuron 83, 975-988 (2014).

50. Lee, Y. I. Developmental neuromuscular synapse elimination: activitydependence and potential downstream effector mechanisms. Neurosci. Lett. 718, 134724 (2020)

51. Gillingwater, T. H. \& Ribchester, R. R. The relationship of neuromuscular synapse elimination to synaptic degeneration and pathology: insights from Wld ${ }^{5}$ and other mutant mice. J. Neurocytol. 32, 863-881 (2003).

52. Son, Y. J. \& Thompson, W. J. Nerve sprouting in muscle is induced and guided by processes extended by Schwann cells. Neuron 14, 133-141 (1995).

53. Orr, B. O. et al. Presynaptic homeostasis opposes disease progression in mouse models of ALS-like degeneration: evidence for homeostatic neuroprotection. Neuron 107, 95-111 (2020).

54. Ayoob, J. C., Terman, J. R. \& Kolodkin, A. L. Drosophila Plexin B is a Sema-2a receptor required for axon guidance. Development 133, 2125-2135 (2006).

55. Orr, B. O., Fetter, R. D. \& Davis, G. W. Retrograde semaphorin-plexin signalling drives homeostatic synaptic plasticity. Nature 550, 109-113 (2017).

56. Gonzalez, M. et al. Disruption of TrkB-mediated signaling induces disassembly of postsynaptic receptor clusters at neuromuscular junctions. Neuron 24, 567-583 (1999).

57. Garcia, N. et al. Involvement of brain-derived neurotrophic factor (BDNF) in the functional elimination of synaptic contacts at polyinnervated neuromuscular synapses during development. J. Neurosci. Res. 88, 1406-1419 (2010).

58. Je, H. S. et al. Role of pro-brain-derived neurotrophic factor (proBDNF) to mature BDNF conversion in activity-dependent competition at developing neuromuscular synapses. Proc. Natl Acad. Sci. USA 109, 15924-15929 (2012).

59. Nadal, L. et al. Presynaptic muscarinic acetylcholine autoreceptors (M1, M2 and M4 subtypes), adenosine receptors (A1 and A2A) and tropomyosin-related kinase $B$ receptor (TrkB) modulate the developmental synapse elimination process at the neuromuscular junction. Mol. Brain 9, 67 (2016).

60. Katz, E., Protti, D. A., Ferro, P. A., Rosato Siri, M. D. \& Uchitel, O. D. Effects of Ca ${ }^{2+}$ channel blocker neurotoxins on transmitter release and presynaptic currents at the mouse neuromuscular junction. Br. J. Pharm. 121, 1531-1540 (1997).

61. Santafé, M. M., Garcia, N., Lanuza, M. A., Uchitel, O. D. \& Tomás, J. Calcium channels coupled to neurotransmitter release at dually innervated neuromuscular junctions in the newborn rat. Neuroscience 102, 697-708 (2001).

62. Mantilla, C. B., Zhan, W.-Z. \& Sieck, G. C. Neurotrophins improve neuromuscular transmission in the adult rat diaphragm. Muscle Nerve 29, 381-386 (2004).

63. Dombert, B. et al. BDNF/trkB induction of calcium transients through $\mathrm{Ca}_{\mathbf{2}} 2.2$ calcium channels in motoneurons corresponds to F-actin assembly and growth cone formation on $\beta 2$-chain laminin (221). Front. Mol. Neurosci. 10, 346 (2017).

64. Schmieg, N., Menendez, G., Schiavo, G. \& Terenzio, M. Signalling endosomes in axonal transport: travel updates on the molecular highway. Semin. Cell Dev. Biol. 27, 32-43 (2014).

65. Villarroel-Campos, D., Schiavo, G. \& Lazo, O. M. The many disguises of the signalling endosome. FEBS Lett. 592, 3615-3632 (2018).

66. Mo, Z. et al. Aberrant GlyRS-HDAC6 interaction linked to axonal transport deficits in Charcot-Marie-Tooth neuropathy. Nat. Commun. 9, 1007 (2018).

67. Deinhardt, K. et al. Rab5 and Rab7 control endocytic sorting along the axonal retrograde transport pathway. Neuron 52, 293-305 (2006).

68. Puls, I. et al. Mutant dynactin in motor neuron disease. Nat. Genet. 33, 455-456 (2003).

69. Verhoeven, $\mathrm{K}$. et al. Mutations in the small GTP-ase late endosomal protein RAB7 cause Charcot-Marie-Tooth type 2B neuropathy. Am. J. Hum. Genet. 72 , 722-727 (2003).

70. Fellows, A. D., Rhymes, E. R., Gibbs, K. L., Greensmith, L. \& Schiavo, G. IGF1R regulates retrograde axonal transport of signalling endosomes in motor neurons. EMBO Rep. 21, e49129 (2020).

71. Wang, $T$. et al. Flux of signalling endosomes undergoing axonal retrograde transport is encoded by presynaptic activity and TrkB. Nat. Commun. 7, 12976 (2016).

72. Allodi, I. et al. Differential neuronal vulnerability identifies IGF-2 as a protective factor in ALS. Sci. Rep. 6, 25960 (2016).

73. Pathak, A., Clark, S., Bronfman, F. C., Deppmann, C. D. \& Carter, B. D. Longdistance regressive signaling in neural development and disease. Wiley Interdiscip. Rev. Dev. Biol. e382 (2020). https://doi.org/10.1002/wdev.382

74. Mantilla, C. B. et al. TrkB kinase activity maintains synaptic function and structural integrity at adult neuromuscular junctions. J. Appl. Physiol. 117 910-920 (2014) 